\title{
THE MAGNETOSPHERE OF THE ULTRACOOL DWARF DENIS 1048-3956
}

\author{
V. Ravi ${ }^{1,5,6}$, G. Hallinan ${ }^{2,7,8}$, G. HobBs ${ }^{3}$, AND D. J. Champion ${ }^{4}$ \\ ${ }^{1}$ Research School of Astronomy and Astrophysics, ANU, Mt. Stromlo Observatory, Weston ACT 2611, Australia \\ 2 National Radio Astronomy Observatory, 520 Edgemont Road, Charlottesville, VA 22903, USA \\ ${ }^{3}$ CSIRO Astronomy and Space Sciences, Australia Telescope National Facility, P.O. Box 76, Epping NSW 1710, Australia \\ ${ }^{4}$ Max-Planck-Institut für Radioastronomie, Auf dem Hügel 69, 53121, Bonn, Germany \\ Received 2010 September 21; accepted 2011 May 4; published 2011 June 3
}

\begin{abstract}
Ultracool dwarfs, the least-massive contributors to the stellar mass function, exhibit striking magnetic properties that are inconsistent with trends for more massive stars. Here, we present the widest-band radio observations to date of an ultracool dwarf, DENIS-P J104814.9-395604, in four $2 \mathrm{GHz}$ bandwidths between wavelengths of $1 \mathrm{~cm}$ and $10 \mathrm{~cm}$. These data were obtained with the Australia Telescope Compact Array using the new Compact Array Broadband Backend instrument. We detected a stable negatively sloped power-law spectrum in total intensity, with spectral index $\alpha=1.71 \pm 0.09$. Circular polarization fractions between 0.25 and 0.4 were found at the low-frequency end of our detection band. We interpret these results as indicative of gyrosynchrotron emission. We suggest that the radio emission originates from beyond the corotation radius, $R_{C}$, of the star. Adopting this model, we find $R_{C}$ between $1.2 R_{*}$ and $2.9 R_{*}$, and a non-thermal electron density and magnetic field strength between $10^{5}$ and $10^{7.2} \mathrm{~cm}^{-3}$ and between 70 and $260 \mathrm{G}$, respectively, at $R_{C}$. The model accounts for the violation of the Güdel-Benz relation between X-ray and radio luminosities of low-mass stars by DENIS-P J104814.9-395604.
\end{abstract}

Key words: radio continuum: stars - stars: individual (DENIS-P J104814.9-395604) - stars: low-mass - stars: magnetic field

\section{INTRODUCTION}

Ultracool dwarfs (UDs), with spectral types M7 and cooler, are the coolest stars and include the least-massive hydrogenburning objects and brown dwarfs (Bessell \& Stringfellow 1993). The interiors of ultracool dwarfs should be fully convective. Observations of magnetic activity tracers (e.g., Berger et al. 2010), direct polarimetric magnetic field strength measurements (e.g., Reiners \& Basri 2007), and magnetic tomography (Morin et al. 2008, 2010; Donati et al. 2008) have revealed vastly different magnetic properties for such fully convective stars compared with more massive solar-type stars.

$\mathrm{X}$-ray and radio emission are tracers of magnetic activity in cool stars. X-ray emission is associated with thermal bremsstrahlung from coronae (Rosner et al. 1985), possibly heated through thermal dissipation of accelerated, energetic electrons (Güdel \& Benz 1993). These electrons also radiate at radio wavelengths, as evinced by a fixed ratio of X-ray to radio luminosities for several classes of active cool stars (the Güdel-Benz relation, Güdel \& Benz 1993). For radio frequencies between $5 \mathrm{GHz}$ and $8 \mathrm{GHz}$, the radio luminosities per unit frequency, $L_{R}$, and the X-ray luminosities, $L_{X}$, are related as $L_{X} / L_{R} \sim 10^{15.5 \pm 0.5} \mathrm{~Hz}$. This relation appears largely independent of a variety of stellar properties, including rotation for periods greater than $\sim 12 \mathrm{hr}$, binarity, spectral class, and photospheric activity, and applies to both variable and quiescent emission phenomena. Phenomenologically, the Güdel-Benz relation implies that magnetic activity, as manifested in coronal

\footnotetext{
5 Also at CSIRO Astronomy and Space Sciences, Australia Telescope National Facility, P.O. Box 76, Epping NSW 1710, Australia.

6 Current address: School of Physics, University of Melbourne, Parkville, VIC 3010, Australia.

7 Also at Department of Astronomy, University of California, Berkeley, CA 94720, USA.

8 G. Hallinan is a Jansky Fellow of the National Radio Astronomy Observatory.
}

heating, results in a non-thermal electron density that is proportional to the degree of activity.

Established trends for cool stars linking magnetic activity, magnetic field strengths and structures, and stellar rotation do not apply to UDs. The ratios of $L_{X}$ to the bolometric luminosities, $L_{\text {bol }}$, for UDs are below those expected from trends for more massive cool stars (Berger et al. 2010). Remarkably, the radio luminosities exhibit an opposite trend, increasing relative to $L_{\text {bol }}$ for UDs (Berger 2006; Berger et al. 2010). The sample of radio-loud UDs includes late-M dwarfs and L dwarfs; no T dwarfs have yet been detected at radio wavelengths (e.g., Berger 2006). All radio-loud UDs violate the Güdel-Benz relation. For their sample of radio-loud UDs, Berger et al. (2010) find $\log \left(L_{X} / L_{R}\right) \sim 14$ for late-M dwarfs, and $\log \left(L_{X} / L_{R}\right) \sim 12$ for cooler dwarfs. No clear trends have been identified between the radio luminosities of UDs and stellar properties, such as rotation and magnetic field strength (Berger et al. 2010).

Radio emission from UDs is variable on timescales of years, hours, and minutes. Some UDs have radio light curves that are periodic on the rotation periods of a few hours (Hallinan et al. 2006, 2007, 2008; Berger et al. 2009). These light curves are either characterized by modulations (e.g., Hallinan et al. 2006) or by short duty-cycle peaks lasting for a few minutes (e.g., Hallinan et al. 2007). Other UDs exhibit isolated flares when otherwise radio loud, also on few-minute timescales (e.g., Burgasser \& Putman 2005, hereafter BP05). The quickly varying emission is generally $100 \%$ circularly polarized (e.g., Hallinan et al. 2008). In addition, the types of radio emission observed from UDs change on timescales of years (Osten et al. 2009), varying between undetectable, quiescent, and periodic. The variability of UD radio emission characteristics makes it hard to identify unbiased radio-loud samples for population studies.

Radio observations of UDs provide significant insight into conditions in UD magnetospheres. In this Letter, we present the widest-band radio observations yet reported for the 
Table 1

Details of Observations

\begin{tabular}{|c|c|c|c|}
\hline Observing Date & Center Frequencies $(\mathrm{GHz})$ & Start and End Times (UT) & Synthesized Beam Sizes \\
\hline 2009 Aug 9 and 10 & 18,24 & $21: 10,06: 10$ & $1^{\prime \prime} .2 \times 0^{\prime \prime} .76,0^{\prime \prime} .90 \times 0^{\prime \prime} .57$ \\
\hline 2009 Aug 11 & 18,24 & $03: 25,07: 45$ & $3^{\prime \prime} .1 \times 0^{\prime \prime} .80,2^{\prime \prime} .34 \times 0^{\prime \prime} .60$ \\
\hline 2009 Aug 15 & $5.5,9$ & $02: 30,07: 20$ & $7^{\prime \prime} .5 \times 2^{\prime \prime} .0,4^{\prime \prime} .6 \times 1^{\prime \prime} .2$ \\
\hline
\end{tabular}

UD DENIS-P J104814.9-395604 (hereafter DENIS1048), using the new Compact Array Broadband Backend (Wilson et al. 2011) at the Australia Telescope Compact Array (ATCA; Frater et al. 1992). We propose a magnetospheric model which accounts for the violation of the Güdel-Benz relation. This model could provide interesting insights into the magnetic field and plasma environments of these enigmatic stars.

\section{OBSERVATIONS AND DATA ANALYSIS}

The target source, DENIS1048, was one of seven Southern late-M and L dwarfs observed by BP05 with the ATCA in the $3 \mathrm{~cm}$ and $6 \mathrm{~cm}$ bands. BP05 reported a quiescent flux density of $0.14 \pm 0.04 \mathrm{mJy}$ at $6 \mathrm{~cm}$, as well as a $4-5$ minute flare in each band, separated by $\sim 10$ minutes with a peak flux density of $30 \mathrm{mJy}$ at $3 \mathrm{~cm}$. DENIS1048 (spectral classification M8.5; Henry et al. 2004) was identified as a UD in the DENIS survey (Epchtein et al. 1997) by Delfosse et al. (2001) and, at a distance of $4.00 \pm 0.03 \mathrm{pc}$ (Costa et al. 2005), is one of the closest known stars. A recent spectroscopic study by Martín et al. (2010) shows that it is unlikely to be a brown dwarf. Fuhrmeister \& Schmitt (2004) reported a large optical flare and a fast projected rotation velocity of $v \sin i=25 \pm 2 \mathrm{~km} \mathrm{~s}^{-1}$. While $\mathrm{H} \alpha$ emission was detected by Delfosse et al. (2001), Schmitt \& Liefke (2004) found no X-ray emission with an upper limit of $2 \times 10^{26} \mathrm{erg} \mathrm{s}^{-1}$ from the ROSAT All-Sky Survey catalog. Reiners \& Basri (2010) found an average line-of-sight magnetic field strength of $2300 \pm 400 \mathrm{G}$ using measurements of Zeeman broadening in FeH absorption lines.

We observed DENIS1048 with the ATCA on 2009 August 10 and 11 in the $1.2 \mathrm{~cm}$ band, and simultaneously in the 3 and $6 \mathrm{~cm}$ bands on 2009 August 15. The six 22 m ATCA antennas were placed in an extended configuration in order to maximize pointsource sensitivity. Baseline lengths ranged between $300 \mathrm{~m}$ and $6000 \mathrm{~m}$, corresponding to resolutions between approximately $40^{\prime \prime}$ and $2^{\prime \prime}$ at $6 \mathrm{~cm}$. Visibility measurements for all baselines were recorded in two $2.048 \mathrm{GHz}$ bands per Stokes polarization with $1 \mathrm{MHz}$ frequency resolution. The visibilities were integrated over $10 \mathrm{~s}$ intervals. Details of the observations are given in Table 1.

We reduced the data using the MIRIAD software package (Sault et al. 1995). Standard calibrations were performed using observations of the ATCA primary calibrator PKS B1934-638 on each day and frequent observations of a radio galaxy, PKS B1104-445, separated by $6^{\circ}$ from DENIS1048. Multi-frequency synthesis total-intensity images were produced in sub-bands of $256 \mathrm{MHz}$ for the $6 \mathrm{~cm}$ and $3 \mathrm{~cm}$ observations, and in each $2 \mathrm{GHz}$ sub-band for the $1.2 \mathrm{~cm}$ observations. We detected DENIS1048 as a point source in all images, except that formed from the $24 \mathrm{GHz}$ data. The measured position of right ascension: $10 \mathrm{~h}, 48 \mathrm{~m}, 13.58 \mathrm{~s}$ ( $\pm 0.03 \mathrm{~s})$, declination: $-39^{\circ}, 56^{\prime}, 16^{\prime \prime} .0\left( \pm 0^{\prime} .5\right)$ is offset from the 2 MASS position of DENIS1048 (Cutri et al. 2003) by 15".6, which corresponds to the known proper motion (Deacon et al. 2005). The flux density of DENIS1048 was measured in each sub-band by fitting the restoring Gaussian beams to the images. The beams were at position angles of $19^{\circ}$ for the $6 \mathrm{~cm}$ and $3 \mathrm{~cm}$ data, and $2^{\circ}$ for the $1.2 \mathrm{~cm}$ data, The rms noise levels, $\sigma$, in images made from each sub-band ranged between 30 and $50 \mu \mathrm{Jy}$ for the $6 \mathrm{~cm}$ data, and between 25 and $35 \mu \mathrm{Jy}$ for the $3 \mathrm{~cm}$ data. For both the $18 \mathrm{GHz}$ and $24 \mathrm{GHz}, \sigma=8 \mu \mathrm{Jy}$.

We present the resulting spectrum of DENIS1048 in Figure 1. The Stokes I measurements of the flux density of DENIS1048, $S(\nu)$, at various frequencies $v$ are an excellent fit to a power law, $S(v) \propto v^{-\alpha}$, where $\alpha=1.71 \pm 0.09$.

A similar process was also applied to the Stokes $Q, U$, and $V$ data. While DENIS1048 was not found to have any detectable Stokes $Q$ or $U$ emission, we detected Stokes $V$ emission at frequencies up to $6.5 \mathrm{GHz}$ by combining data in multiple subbands. Circular polarization fractions ranging between 0.25 and 0.4 were found in the $6 \mathrm{~cm}$ band, and $3 \sigma$ upper limits of 0.2 were placed on the linear polarization fractions. The Stokes $V$ flux density measurements are also plotted in Figure 1. An image of the data recorded between $4.576 \mathrm{GHz}$ and $4.988 \mathrm{GHz}$, with Stokes $V$ contours overlayed on a Stokes $I$ gray-scale image, is shown in Figure 2. No significant short-timescale amplitude excursions or periodicities were detected in any sub-band in either the Stokes $I$ or $V$ data.

\section{THE RADIO EMISSION MECHANISM}

We consider two possible radio emission mechanisms for DENIS1048: gyrosynchrotron emission and electron-cyclotron maser (ECM) emission. The spectral shape and amplitude are clearly inconsistent with thermal emission. Gyrosynchrotron and ECM mechanisms are both associated with mildly relativistic, non-thermal electron populations, with energies $>\sim 20 \mathrm{keV}$. Whereas gyrosynchrotron emission is caused by incoherently radiating non-thermal electrons propagating along magnetic field lines, ECM emission is coherent, and requires these electrons to have an anisotropic pitch-angle distribution (Dulk 1985). Gyrosynchrotron emission is characterized by a negatively sloped power-law spectrum at high frequencies, corresponding to optically thin emission, and a positively sloped spectrum at lower frequencies corresponding to optically thick emission. ECM emission, however, occurs at frequencies corresponding to low harmonics of the local cyclotron frequency and does not have a characteristic spectral shape (Melrose 2009).

Time-variable emission from UDs is up to $100 \%$ circularly polarized and tightly beamed, and is hence interpreted as ECM emission (e.g., Hallinan et al. 2008). Gyrosynchrotron emission has been hypothesized by a variety of authors for radio-loud UDs in quiescent states (e.g., BP05; Osten et al. 2006).

The observed radio properties of DENIS1048 are more suggestive of gyrosynchrotron emission than ECM emission for three reasons.

1. The negatively sloped power-law spectrum we observe is expected of optically thin gyrosynchrotron emission, whereas no particular spectral shape is uniquely identified with ECM emission.

2. If the $1.2 \mathrm{~cm}$ emission were caused by an ECM mechanism, the emission would need to originate in a stable region 


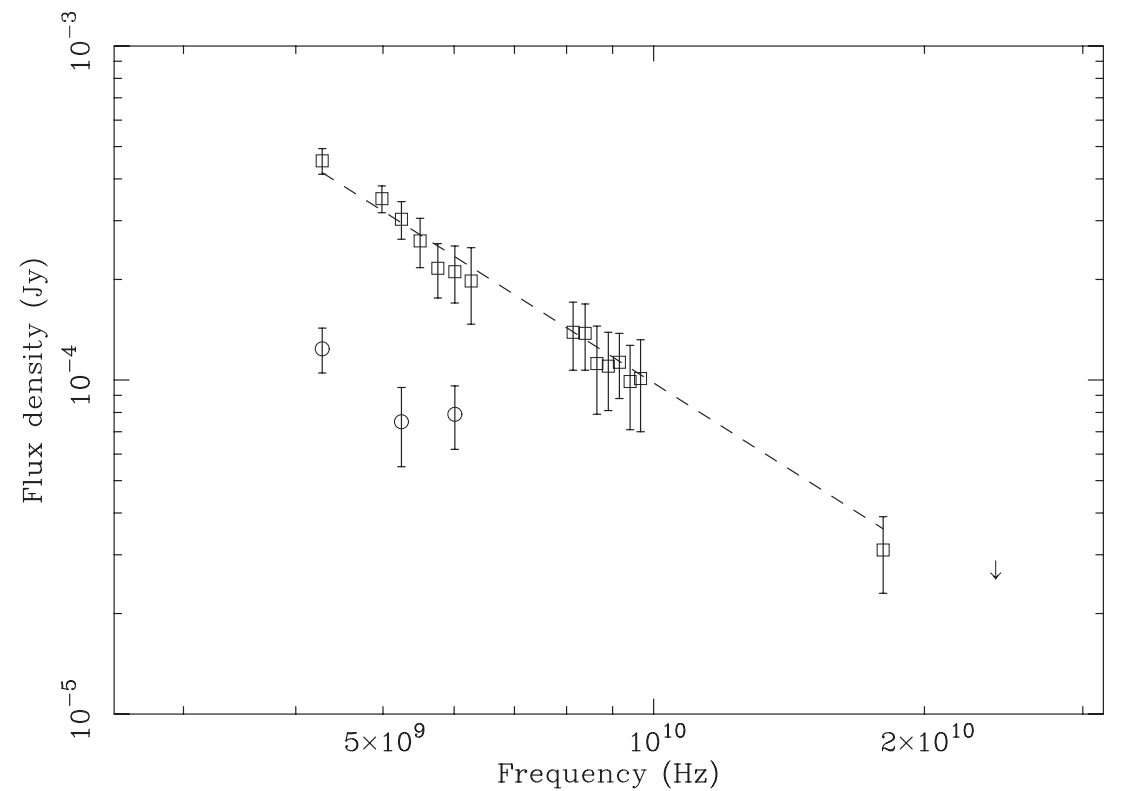

Figure 1. Radio spectrum of DENIS1048 obtained with the ATCA between 2009 August 11 and 15 . The squares are the Stokes $I$ measurements, the circles are the Stokes $V$ measurements, and the arrow indicates the $3 \sigma$ upper limit we place for the $24 \mathrm{GHz}$ emission. The dashed line is the best-fit power law $\left(S(v) \propto v^{-\alpha}\right)$ to the Stokes $I$ spectrum, with index $\alpha=1.71 \pm 0.09$.

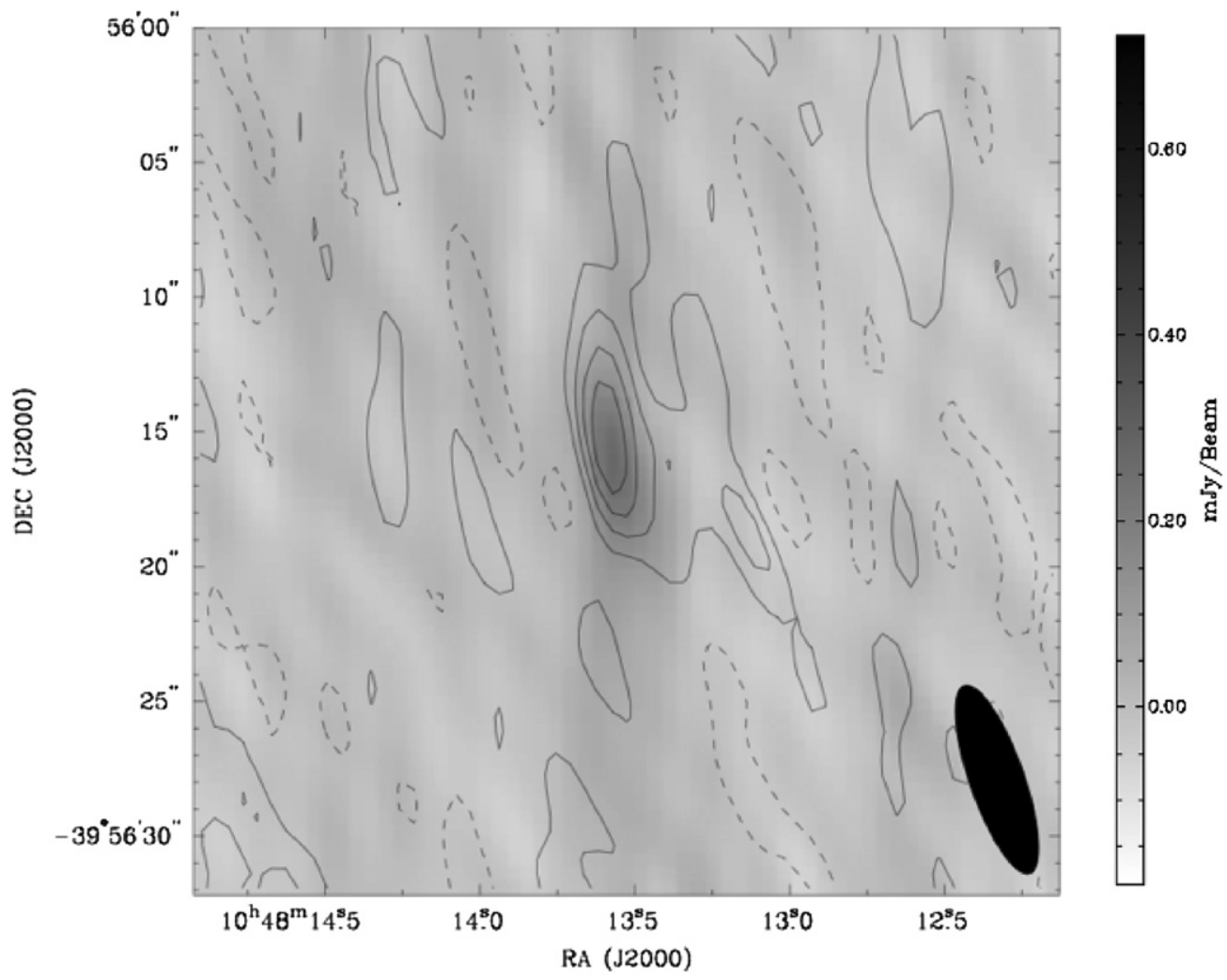

Figure 2. Image of the Stokes I (gray scale) and Stokes $V$ (contours) emission from DENIS1048 made using data at frequencies between 4.576 and $4.988 \mathrm{GHz}$. These data were obtained on 2009 August 15 using the ATCA, with a maximum baseline length of $6 \mathrm{~km}$. The beam used to make the image, shown as a filled ellipse in the bottom-right corner, had a size of $8^{\prime \prime} .7 \times 22^{\prime \prime} 3$ at a position angle of $19^{\circ}$. The Stokes $V$ contour levels are at $-1 \sigma$ (dashed), $1 \sigma, 2 \sigma, 3 \sigma$, and $4 \sigma$, where $\sigma=25 \mu \mathrm{Jy}$. The maximum Stokes $I$ intensity in the field is $370 \mu \mathrm{Jy}$.

with a magnetic field strength of approximately $6.4 \mathrm{kG}$ to be consistent with emission at the fundamental cyclotron frequency. Unless the line of sight is nearly aligned with the rotation axis, such a region must also cover a significant fraction of the stellar surface, which is unlikely (Reiners \& Basri 2010).
3. The lack of significant modulation is marginally inconsistent with the tight beaming of ECM emission.

While we cannot conclusively rule out an ECM emission mechanism, we interpret the radio emission that we observe from DENIS1048 as optically thin gyrosynchrotron emission. In this interpretation, the large observed Stokes $V$ 
Table 2

Constrained Ranges for the Model Parameters

\begin{tabular}{lcc}
\hline \hline$R_{C} / R_{J}$ & $N_{e}\left(\mathrm{~cm}^{-3}\right)$ & $B_{C}(\mathrm{G})$ \\
\hline $1.2-2.9$ & $10^{5}-10^{7.2}$ & $70-260$
\end{tabular}

fractions are consistent with a strong line-of-sight magnetic field component. Furthermore, the number of radiating electrons, $N(E)$, per unit energy, $E$, can be written as a power law, $N(E) \propto E^{-\delta}$, where $\delta=(1.22-\alpha) / 0.9$ (Dulk 1985). We find $\delta=3.26 \pm 0.09$, consistent with expected values between -2 and -7 for gyrosynchrotron-emitting electrons (Dulk 1985).

\section{A MODEL FOR THE MAGNETOSPHERE}

Assuming $L_{X}<2 \times 10^{26} \mathrm{erg} \mathrm{s}^{-1}$ after Schmitt \& Liefke (2004) for DENIS1048, the Güdel-Benz relation implies an average radio flux density between $5 \mathrm{GHz}$ and $8 \mathrm{GHz}$ of less than $42 \mu \mathrm{Jy}$. At the lowest observing frequency, the measured flux density is an order of magnitude greater.

We propose a model for the magnetosphere of DENIS1048 that accounts for the violation of the Güdel-Benz relation. For samples of fast-rotating $\mathrm{M}$ dwarfs, an observed decrease in $L_{X} / L_{\text {bol }}$ with rotation period (Berger et al. 2010; Jeffries et al. 2011 ) is interpreted as evidence for the decoupling of hot coronal plasma beyond a corotation radius, $R_{C}$. We hypothesize that this effect does not reduce $L_{R} / L_{\mathrm{bol}}$. We further suggest that

1. $R_{C}$ represents the Alfvèn radius of the star, and the magnetic field structure beyond $R_{C}$ is approximately radial and dominated by the outflow of non-thermal electrons;

2. electron acceleration occurs at $R_{C}$, possibly through magnetic reconnection (Zweibel \& Yamada 2009).

In this model, the gyrosynchrotron emission we observe originates from non-thermal electrons streaming radially outward from $R_{C}$. A radial magnetic field structure in this region is justified by the lack of significant variability in the radio light curve.

The radio emission is characterized by three parameters: $R_{C}$, the total non-thermal electron density, $N_{e}$, at $R_{C}$, and the radial magnetic field strength, $B_{C}$, at $R_{C}$. We attempted to uniquely measure these parameters through a fit to the measured spectrum of DENIS1048, using expressions from Dulk (1985) for the total and circularly polarized intensities of gyrosynchrotron emission. A large number of parameter combinations were found to fit the data. Two assumptions, however, allowed the free parameters to be constrained within the ranges given in Table 2. First, we assumed that the spectral peak of the gyrosynchrotron emission (Dulk 1985) did not lie within the spectral band, as justified by the regularity of the observed power-law spectrum. We also limited $B_{C}<2700 /\left(R_{C}-R_{*}\right)^{3} \mathrm{G}$ in the dipole approximation, where $2700 \mathrm{G}$ is the upper limit on the surface magnetic field strength placed by Reiners \& Basri (2010). The stellar radius, $R_{*}$, is further assumed (after, e.g., Benz et al. 1995; Burrows et al. 2001; Burgasser \& Putman 2005; Osten et al. 2009) to be equivalent to a Jupiter radius.

\section{CONCLUSIONS AND FUTURE WORK}

We have utilized the unprecedented frequency coverage of the upgraded ATCA to characterize the radio spectrum of the UD DENIS1048. Between $4.5 \mathrm{GHz}$ and $24 \mathrm{GHz}$, the spectrum follows a strikingly regular power law with index $\alpha=$ $1.71 \pm 0.09$. This spectral shape, the lack of time variability, and circular polarization fractions between 0.25 and 0.4 in the $6 \mathrm{~cm}$ band are suggestive of a gyrosynchrotron mechanism for the radio emission.

Further work is required to improve and test the model we present. An observation of a peak frequency in the spectrum of DENIS1048 would break the degeneracy between the free parameters. It is possible that the model predicts correlations between radio emission characteristics of UDs and the stellar parameters. Such correlations are yet to be investigated. Finally, the model does not account for radio emission other than from a gyrosynchrotron mechanism, or for time-variable emission. Efforts are under way to model UDs in such states (e.g., Yu et al. 2010).

We thank the ATCA staff and duty astronomers for their valuable assistance with the observations reported here. The Australia Telescope Compact Array is part of the Australia Telescope which is funded by the Commonwealth of Australia for operation as a National Facility managed by CSIRO. This research has made use of the SIMBAD database, operated at CDS, Strasbourg, France. G.H. is supported by an Australian Research Council QEII Fellowship (project no. DP0878388). The National Radio Astronomy Observatory is a facility of the National Science Foundation operated under cooperative agreement by Associated Universities, Inc.

\section{REFERENCES}

Benz, A. O., Alef, W., \& Güdel, M. 1995, A\&A, 298, 187

Berger, E. 2006, ApJ, 648, 629

Berger, E., et al. 2009, ApJ, 695, 310

Berger, E., et al. 2010, ApJ, 709, 332

Bessell, M. S., \& Stringfellow, G. S. 1993, ARA\&A, 31, 433

Burgasser, A. J., \& Putman, M. E. 2005, ApJ, 626, 486

Burrows, A., Hubbard, W. B., Lunine, J. I., \& Liebert, J. 2001, Rev. Mod. Phys., 73,719

Costa, E., Méndez, R. A., Jao, W.-C., Henry, T. J., Subasavage, J. P., Brown, M. A., Ianna, P. A., \& Bartlett, J. 2005, AJ, 130, 337

Cutri, R. M., et al. 2003, The IRSA 2MASS All-Sky Point Source Catalog, NASA/IPAC Infrared Science Archive (Pasadena, CA: Caltech)

Deacon, N. R., Hambly, N. C., \& Cooke, J. A. 2005, A\&A, 435, 363

Delfosse, X., et al. 2001, A\&A, 366, L13

Donati, J.-F., et al. 2008, MNRAS, 390, 545

Dulk, G. A. 1985, ARA\&A, 23, 169

Epchtein, N., et al. 1997, Messenger, 87, 27

Frater, R. H., Brooks, J. W., \& Whiteoak, J. B. 1992, J. Electr. Electron. Eng. Aust., 12, 103

Fuhrmeister, B., \& Schmitt, J. H. M. M. 2004, A\&A, 420, 1079

Güdel, M., \& Benz, A. O. 1993, ApJ, 405, L63

Hallinan, G., Antonova, A., Doyle, J. G., Bourke, S., Brisken, W. F., \& Golden, A. 2006, ApJ, 653, 690

Hallinan, G., Antonova, A., Doyle, J. G., Bourke, S., Lane, C., \& Golden, A. 2008, ApJ, 684, 644

Hallinan, G., et al. 2007, ApJ, 663, L25

Henry, T. J., Subasavage, J. P., Brown, M. A., Beaulieu, T. D., Jao, W.-C., \& Hambly, N. C. 2004, AJ, 128, 2460

Jeffries, R. D., Jackson, R. J., Briggs, K. R., Evans, P. A., \& Pye, J. P. 2011, MNRAS, 411, 2099

Martín, E. L., et al. 2010, A\&A, 517, A53

Melrose, D. B. 2009, in IAU Symp. 257, Universal Heliophysical Processes, ed. N. Gopalswamy \& D. F. Webb (Cambridge: Cambridge Univ. Press), 305

Morin, J., et al. 2008, MNRAS, 390, 567

Morin, J., Donati, J.-F., Petit, P., Delfosse, X., Forveille, T., \& Jardine, M. M. 2010, MNRAS, 407, 2269

Osten, R. A., Hawley, S. L., Bastian, T. S., \& Reid, I. N. 2006, ApJ, 637, 518

Osten, R. A., Phan-Bao, N., Hawley, S. L., Reid, I. N., \& Ojha, R. 2009, ApJ, 700,1750

Reiners, A., \& Basri, G. 2007, ApJ, 656, 1121 
Reiners, A., \& Basri, G. 2010, ApJ, 710, 924

Rosner, R., Golub, L., \& Vaiana, G. S. 1985, ARA\&A, 23, 413

Sault, R. J., Teuben, P. J., \& Wright, M. C. H. 1995, in ASP Conf. Ser. 77 Astronomical Data Analysis Software and Systems IV, ed. R. A. Shaw, H. E. Payne, \& J. J. E. Hayes (San Francisco, CA: ASP), 433
Schmitt, J. H. M. M., \& Liefke, C. 2004, A\&A, 417, 651

Wilson, W. E., et al. 2011, MNRAS, in press (arXiv:1105.3532)

Yu, S., Hallinan, G., Doyle, J. G., MacKinnon, A. L., Antonova, A., Kuznetsov, A., Golden, A., \& Zhang, Z. H. 2011, A\&A, 525, A39

Zweibel, E. G., \& Yamada, M. 2009, ARA\&A, 47, 291 